\title{
Reversible and Embedded Watermarking of Medical Images for Telemedicine
}

\author{
Chung-Yen Su, Jeng-Ji Huang, Che-Yang Shih, Yu-Tang Chen \\ Department of Electrical Engineering \\ National Taiwan Normal University, Taiwan \\ scy@ntnu.edu.tw, hjj2005@ntnu.edu.tw,60275003H@ntnu.edu.tw,60270026H@ntnu.edu.tw
}

\begin{abstract}
In this paper, we propose a new reversible watermarking of medical images for the applications in telemedicine. By using a bit-stream insertion scheme, the patient's information can be treated as a watermark and can be embedded into the bit-stream of a cover image for remote transmission. The proposed method simplifies the design of traditional image coding after a reversible watermarking. Experimental results show that the compression ratio can be achieved up to 3.025. The watermarking capacity can be obtained over 0.75 bpp for some common images. In addition, the watermark can be extracted exactly and the cover image can be reconstructed either in a lossless way or a lossy way. The obtained results also show the improvement with respect to previous works.
\end{abstract}

Keywords-integer wavelet transform; lossless compression; reversible watermarking; SPIHT

\section{INTRODUCTION}

Telemedicine can be used to provide clinical health care at a distance. In telemedicine, medical images are transmitted to physician or specialist through the internet or mobile phones. Hence, special security and confidentiality should be taken into consideration. Digital watermarking is a technique for data hiding, which can be used to increase the level of security and to verify authenticity. In the traditional digital watermarking, the hospital logo can be used as the watermark to provide copyright protection. However, to improve the diagnostic results, patient's information, including heart beat rate and body temperature can also be added to the images as the watermark, as shown in Fig. 1.

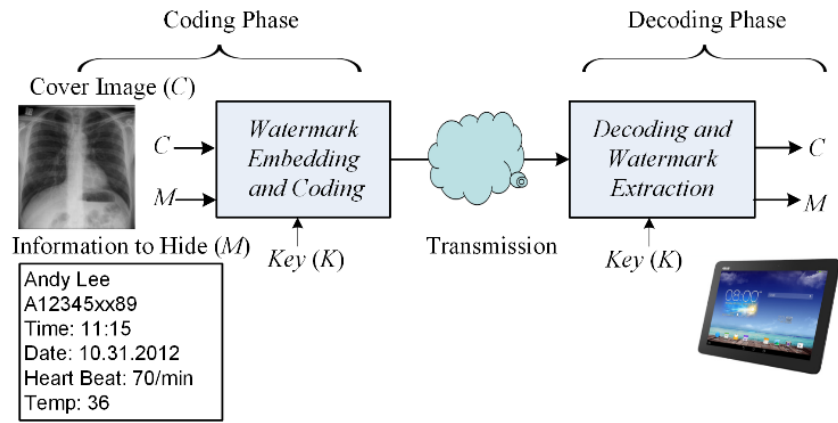

Fig. 1. General structure of the proposed reversible watermarking for telemedicine
In literature, many digital watermarking methods have been proposed in [1]. But, reversible watermarking methods are preferred for the medical images. This is because reversible watermarking gives a zero-distortion cover image, which can be used to avoid a wrong diagnosis.

Typical reversible watermarking methods are based on either difference expansion [2] or histogram shifting [3]. Both of them can be implemented in spatial domain or frequency domain. In [2], Tian first introduces the technique of difference expansion and shows a low distortion data embedding. In [4], the difference expansion technique is applied to the high-frequency subbands of integer wavelet transform (IWT) in order to increase the peak-signal to noise ratio (PSNR) of the watermarked images. In [5], a discrete wavelet transform ordering scheme is proposed to increase the watermark embedding capacity. To further increase the capacity, an effective way is to define a region of interest (ROI). The watermark is embedded with different strength in ROI and non-ROI. In [6], an interactive ROI scheme is proposed and the watermark is embedded in the non-ROI. In [7], an automatic division of ROI and non-ROI is presented and the watermark is embedded based on the block energy difference. Although difference expansion based methods are effective for achieving a reversible watermarking, it is generally required to store the location map to retrieve the original image. To reduce the requirement of location map, a possible way is to use the scheme of histogram shifting. For example, an intelligent reversible watermarking is proposed in [8], which is based on histogram shifting and the genetic algorithm. An improved histogram shifting based on the selection of multiple zero points can be referred to [9]. The penalty of histogram shifting is that the watermark capacity is reduced.

For a remote transmission, compression is always required to reduce the storage size of image or to speed up the transmission. However, the aforementioned methods do not address the effect of compression. After the watermarking, the used compression algorithm should be well designed. Otherwise, the watermark may be disappeared. Due to the location map or to increase the watermarking capacity, it is difficult to modify the coding methods, like JPEG2000 or H.264 intra-mode coder, to achieve a reversible watermarking in a single transmitted file.

This work was partly supported by the National Science Council under

107_-2018_F_nח2_nก1_MV? 
In this paper, a reversible and embedded watermarking is proposed. The basic concept of the proposed method is based on the bit-stream insertion scheme. We integrate the processes of both the lossless coding and the watermark embedding for the goal of remote transmission. The lossless coding is based on the technique of set partitioning in hierarchical trees (SPIHT) [10]. The bit-stream of watermark is generated in a predictive way and is inserted into the SPIHT coding. The proposed method does not require a location map because the locations of watermarked pixels are denoted in the coding process. Moreover, the proposed method can embed watermark bits into the same coefficient many times, and thus can achieve the watermarking capacity over $0.75 \mathrm{bpp}$, which is the upper bound for many DWT based methods.

The rest of the paper is organized as follows. Section 2 briefly describes the overview of our method. In Section 3, we detail the proposed method in the coding phase. Experimental results are illustrated in Section 4. Finally, we make some concluding remarks in Section 5.

\section{OVERVIEW OF OUR METHOD}

Figure 2 shows the flow chart of the proposed watermark embedding process. We modify the SPIHT coding in order to embed the patient information (as watermark). The cover medical image is transformed by the integer wavelet transform. The neighbor pixels of patient information are predicted to generate the watermark bit-stream with less correlation. The bit-stream is then sent to the modified SPIHT coder. The watermark embedding starts when the embedding control is enabled. The embedding control is as a key for watermark extraction. It is used to increase the security of cover image. The output of the embedding process is a compressed watermarked image that can be stored in the cloud or can be transmitted to a remote device for decoding.

Figure 3 shows the watermark extraction process of the proposed method. The proposed method is blind. Thus, the cover medical image is not required for the extraction process. Besides, the location map is not needed because the watermark is embedded in the wavelet-tree coding. Another merit of the proposed method is that the decoding algorithm, i.e. the modified SPIHT decoding, can be known at the decoding side.

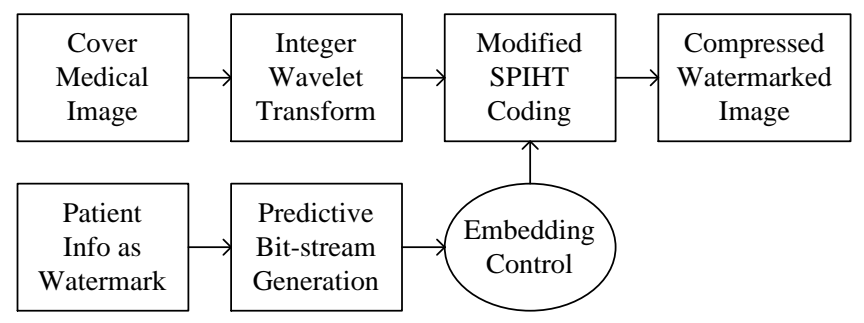

Fig. 2. The flow chart of the proposed watermark embedding process
Even the decoding algorithm is already known, the entire watermark still can not be extracted if we do not know the key of embedding control.

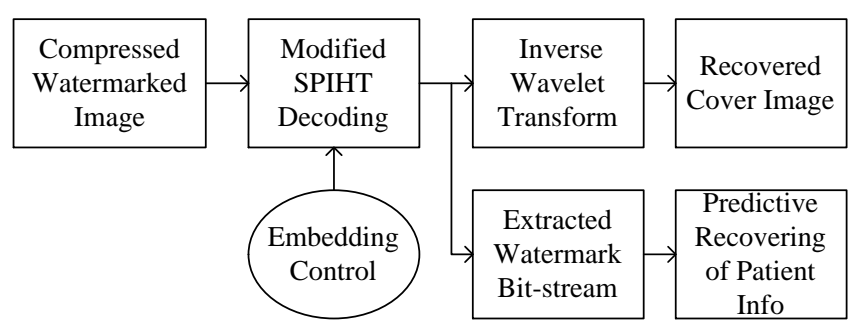

Fig. 3 The flow chart of the proposed watermark extraction process

\section{PROPOSED REVERSIBLE EMBEDDED WATERMARKING}

\section{A. Reversible wavelet transform}

We use the 5-3 reversible wavelet transformation, used in JPEG2000 for lossless image compression, to decompose an image. The reversible transformation is defined using lifting-based filtering. For a row or a column of an image

$$
\ldots p[-3] p[-2] p[-1] p[0] p[1] p[2] p[3] \ldots
$$

The high-pass (HP) and low-pass (LP) wavelet coefficients are shown as follows, respectively.

$\mathrm{HP}: \quad q[2 n+1]=p[2 n+1]-\left\lfloor\frac{p[2 n]+p[2 n+2]}{2}\right\rfloor$

LP: $q[2 n]=p[2 n]+\left\lfloor\frac{q[2 n-1]+q[2 n+1]+2}{4}\right\rfloor$

where $\lfloor x\rfloor$ is the floor operation and the resulting coefficients are of an integer. One scale decomposition is to decompose an image by the reversible transformation horizontally and vertically. A typical three-scale pyramidal decomposition of an image is illustrated in Fig. 4.

\section{B. Modified SPIHT Coding}

We use a modified SPIHT algorithm for image coding and watermark embedding. An embedding control is inserted into the original SPIHT algorithm. We start to embed the watermark bit-stream when the control is enabled. The original SPIHT coding is briefly introduced here. More details of SPIHT can be referred to [10].

We call a node as $C(i, j)$ and an offspring set as $O(i, j)$. In general, $O(i, j)$ involves $\{C(2 i, 2 j), C(2 i, 2 j+1), C(2 i+1,2 j)$, $C(2 i+1,2 j+1)\}$ except the nodes in the highest layer (Layer 4 in Fig. 4) and the lowest layer Layer. A descendant set $D(i$, $j)$ is defined as all the nodes at all finer scales with the same spatial location and of similar orientation. 


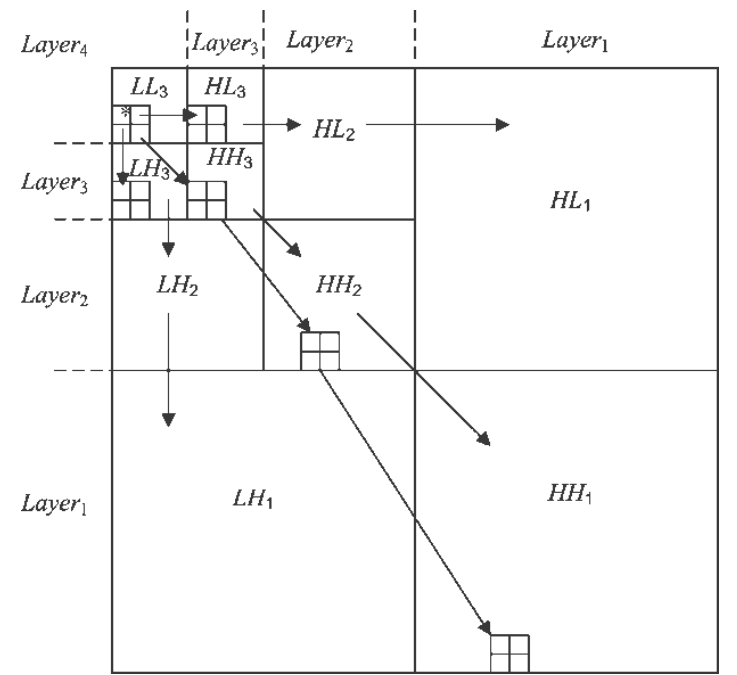

Fig. 4 Example of the three-scale pyramidal decomposition of an image and the tree structure. The SPIHT uses the tree structure, where a parent node has four child nodes except the nodes in Layer 1 and in Layer 4 .

A set $L(i, j)$ is defined as $L(i, j)=D(i, j)-O(i, j)$, and the root set is called $\boldsymbol{H}$. A node or a set is significant if the significant test (3) results in 1.

$S_{n}(X(i, j))=\left\{\begin{array}{l}1, \text { if } \max _{C(k, l) \in X(i, j)}\{|C(k, l)|\} \geq 2^{n} \\ 0, \text { otherwise. }\end{array}\right.$

where $X(i, j)$ denotes $C(i, j), D(i, j)$, or $L(i, j)$. A significant $D(i, j)$ is partitioned into $O(i, j)$ and $L(i, j)$, while a significant $L(i, j)$ is partitioned into $\{D(2 i, 2 j), D(2 i, 2 j+1)$, $D(2 i+1,2 j), D(2 i+1,2 j+1)\}$. The SPIHT algorithm uses three lists for coding, namely list of significant pixels (LSP), list of insignificant pixels (LIP), and list of insignificant sets (LIS). The pseudo code of the algorithm is as follows.

1) Find the maximum value of $n$;

2) Initialize the three lists;

3) Do \{

If $(n==m)$, enable the watermark embedding

Encode each $C(i, j)$ in LIP;

Encode each $D(i, j)$ and $L(i, j)$ in LIS;

Encode each $C(i, j)$ in LSP;

Update LSP;

Update LIS;

Decrease $n$ by 1 ;

\} while $(n>1)$

In the pseudo code, $m$ denotes the bit-plane, in which we start to embed the watermark. The value of $m$ controls the position of the watermark embedded in the resulting bit-stream, and thereby governs the decoded image quality if attacks occur in the transmission pass.

In the proposed coding algorithm, there are several ways to embed the watermark message. In this paper, we examine the following two ways. One is the whole message embedding and the other is the partial message embedded in the entry coding of LIP.

\section{Whole message embedding}

In the way of whole message embedding, the watermark bit-stream is totally embedded before the coding of LIP at the $m$ th bit plane, as shown in Fig. 5. This leads to that the watermark can be extracted exactly even if the bit-stream for the $k$ th bit plane $(k<m)$ is not decoded yet. The larger the value of $m$ is, the nearer to the beginning of the bit-stream the watermark is. Therefore, the watermark can be extracted earlier in a successive transmission. If the extracted watermark shows a wrong information, the transmission can be terminated immediately.

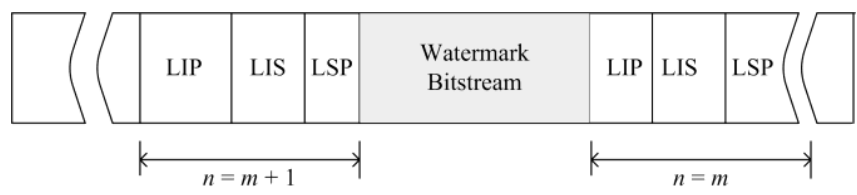

Fig.5 Bit-stream structure in the whole message embedding

\section{Partial message embedded in the entry coding of LIP}

In the way of partial message embedded in the entry coding of LIP, the watermark bit-stream is scattered and is embedded in the bit-stream of entry coding of LIP. Fig. 6 illustrates the bit-stream structure in this way. Since the watermark bit-stream is scattered, it is impossible to reconstruct the watermark completely unless we receive all the pieces of the watermark bit-stream.

In fact, the bit-stream of watermark and the bit-stream of each $C(i, j)$ in LIP can be mixed together to avoid the requirement of a location map. A method to achieve the goal is to embed a watermark bit before coding each $C(i, j)$.

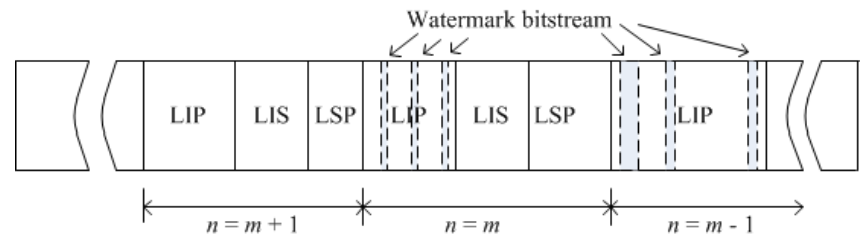

Fig. 6 Bit-stream structure in the partial message embedded in the entry coding of LIP

\section{E. Predictive bit-stream generation of watermark message}

Since there is a high correlation between the neighboring pixels of a watermark, we use a predictive scheme to generate the watermark bit-stream. For each row of the watermark message, the pseudo code of the scheme is as follows.

IF (current_pixel==previous_pixel), THEN Output 0 . ELSE Output 1. 


\section{EXPERIMENTAL RESULTS}

In the experiment, we use nine medical images, as shown in Fig. 7, as cover images. The sizes of the images are $512 \times 512$ (Fig. 7(a)-(g), 600×493 (Fig. 7(h)), and $888 \times 666$ (Fig. 7(i)), respectively. The watermark is a real-time generated patient info with $100 \times 52$ size, which is illustrated in Fig. 8.

The proposed reversible and embedded watermarking is applied to these medical images. In the coding phase, we use the lossless coding. Thus, both the whole message embedding (denoted by W1) and the partial message embedding (denoted by $\mathrm{W} 2$ ) result in the same size of bit-stream. Table I tabulates the compression ratio for each image. We set $m$ to 4 so that only the last 4 bits of the wavelet coefficients are affected by the watermark.

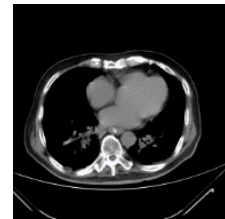

(a)

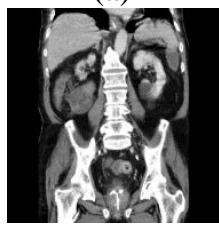

(d)

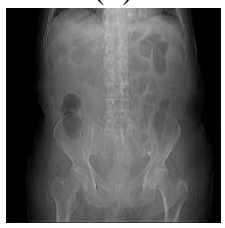

(g)

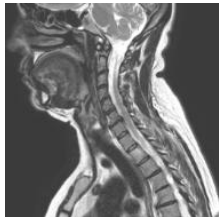

(b)

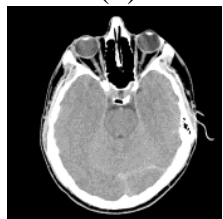

(e)

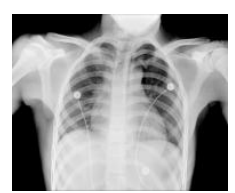

(h)

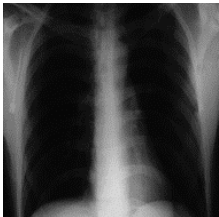

(c)

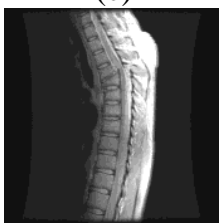

(f)

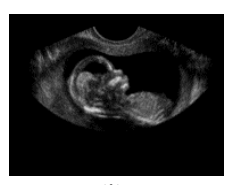

(i)
Fig. 7 Cover images (a)-(i)

張三丰 $\mathrm{t}=36.5 \mathrm{~b}=67$ A123456789

$2014073121: 11: 14$

Fig. 8 The watermark

Table I. Compression ratios of the test images

\begin{tabular}{|c|c|c|c|}
\hline \multirow{4}{*}{$\begin{array}{c}\text { Comp. } \\
\text { ratio }\end{array}$} & Fig.7(a) & Fig. 7(b) & Fig. 7(c) \\
\cline { 2 - 4 } & 4.10 & 2.60 & 2.71 \\
\cline { 2 - 4 } & Fig. 7(d) & Fig.7(e) & Fig.7(f) \\
\cline { 2 - 4 } & 2.69 & 3.27 & 2.92 \\
\cline { 2 - 4 } & Fig. 7(g) & Fig.7(h) & Fig.7(i) \\
\cline { 2 - 4 } & 2 & 2.473 & 5.33 \\
\hline
\end{tabular}

Table II. Experimental results at the conditions of $m=4, \mathrm{~W} 1$ and decoding rate $=0.6 \mathrm{bpp}$

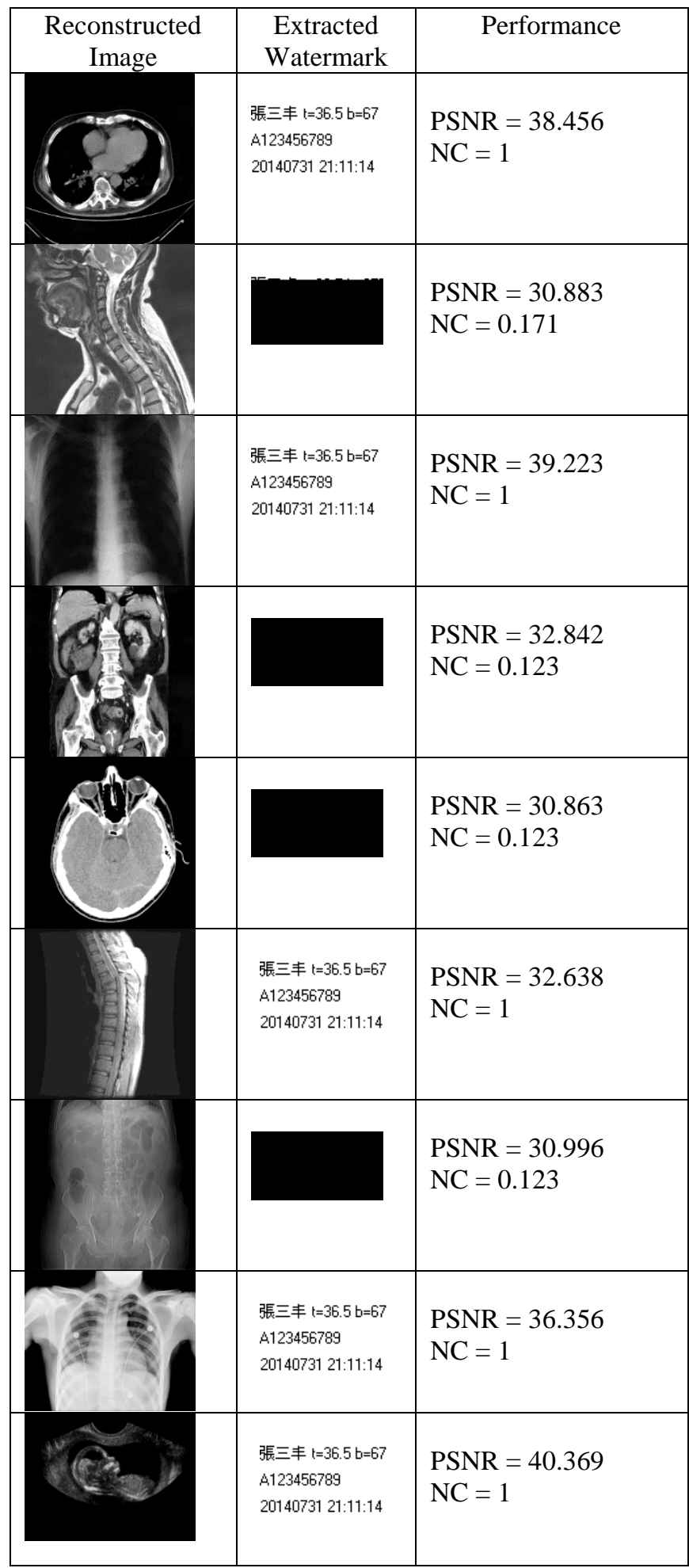

In the decoding phase, we can recover the medical images and the watermark exactly when we use the lossless decoding. However, only part of the medical images and the 
watermark can be recovered when we use a lossy decoding or terminate the bit-stream transmission early.

Table II shows the experimental results at the conditions of using W1 and at decoding rate 0.6 bits per pixel (bpp). As we can see from Table II, the watermark can be extracted completely for the cover images Fig. 7(a), (c), (f), (h) and (i). But, it can not be extracted at all for Figs. 7(d), (e) and (g) and can be extracted only a little for Fig. 7(b). For these images, the decoding rate should be increased so that the watermark can be extracted completely. Note that the reconstructed images herein are not the same as their original version because we use the lossy decoding. To obtain a perfect reconstruction, we need to increase the decoding rate until all the bit-stream is decoded.

We measure the reconstructed image quality by the peak-signal to noise ratio (PSNR). The PSNR is defined by

$$
\text { PSNR }=10 \cdot \log _{10} \frac{255^{2}}{\frac{1}{M N} \sum_{i=0}^{M-1} \sum_{j=0}^{N-1}\left(f_{i j}-\hat{f}_{i j}\right)^{2}}
$$

where $M$ and $N$ are the image width and height, respectively, and $f$ and $\hat{f}$ denote the original image and the recovered image in the decoding phase, respectively. On the other hand, we use the normalized correlation coefficient (NC) to measure the extracted watermark. The NC is defined by

$$
\mathrm{NC}=\frac{1}{W_{h} \times W_{w}} \sum_{i=0}^{W_{h}-1} \sum_{j=0}^{W_{w}-1} w_{i j} \times w_{i j}^{\prime}
$$

where $W_{h}$ and $W_{w}$ separately denote the height and the width of the watermark, and $w$ and $w^{\prime}$ denote the original watermark and the extracted watermark, respectively.

Figure 9 shows the PSNR comparison and the NC comparison between $\mathrm{W} 1$ and W2 for Fig. 7(a). For the bit rates smaller than $0.58 \mathrm{bpp}$, the PSNR values of $\mathrm{W} 1$ and $\mathrm{W} 2$ are the same and so are the NC values. This is because the watermark bit-stream is not inserted yet. For the bit rates between $0.58 \mathrm{bpp}$ and $0.63 \mathrm{bpp}$, W2 results in larger PSNR value and smaller $\mathrm{NC}$ value than $\mathrm{W} 1$ at the same bpp as expected. Therefore, for the quality-priority applications, the partial message embedding W2 is more suitable than the whole message embedding W1. For the bit rates higher than $0.63 \mathrm{bpp}$, the results of $\mathrm{W} 1$ and $\mathrm{W} 2$ are the same again because the watermark bit-stream is already extracted completely.

For the security, we use $m$, which is chosen for hiding the watermark message, to serve as the key to protect the message. Without the key, the extraction message will be wrong in the decoding phase. Moreover, since the generated bit-stream of the modified SPIHT coding is adaptively arithmetically coded in multiple models before transmission, both the watermark and cover image are secure if the models are unknown in the decoding side.

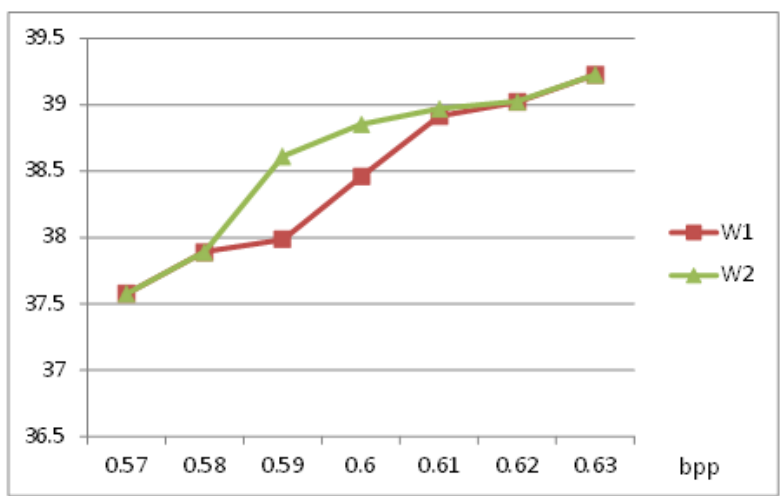

(a)

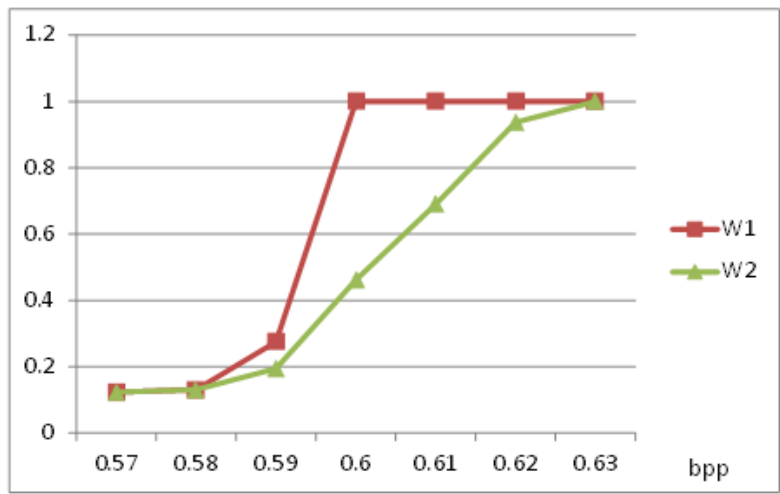

(b)

Fig. 9 (a) PSNR and (b) NC comparison between the whole massage embedding (W1) and the partial message embedding (W2)

\section{CONCLUSION}

In this paper, a new reversible and embedded watermarking by bit-stream insertion is proposed. The proposed method integrates both the watermarking and the image coding together, which results in a single file for effectively remote transmission. The proposed method does not require a location map and can achieve a high watermark capacity. Extensive experiments show the effectiveness of the proposed method.

\section{REFERENCES}

[1] A. Cheddad, J. Condell, K. Curran, P. M. Kevitt, "Digital image steganography: survey and analysis of current methods," Signal Processing, 90, 2010, pp. 727-752.

[2] J. Tian, "Reversible data embedding using a difference expansion," IEEE Trans. on Circuits and Systems for Video Tech., pp. 890-896, Aug. 2003.

[3] G. Xuan, Q. Yao, C. Yang, J. Gao, P. Chai, Y. Q. Shi, Z. Ni, "Lossless data hiding using histogram shifting method based on integer wavelet," in International Workshop on Digital Watermarking, vol. 4283, pp. 323-332, 2006. 
[4] G. Xuan, Y. Q. Shi, C. Yang, Y. Zheng, D. Zou, P. Chai, "Lossless data hiding using integer wavelet transform and threshold embedding technique," in the Proc. IEEE international conference on Multimedia and Expo, pp. 1520-1523, 2005.

[5] A. K. Al-Asmari, A. Salama, A. M. Iliyasu, M. A. Al-Qodah, "A DWT ordering scheme for hiding data in images using pixel value difference," in the Proc. IEEE Eighth International Conf. on Computational Intelligence and Security, pp. 553-557, 2012.

[6] B. L. Gunjal, and S. N. Mali, "ROI based embedded watermarking of medical images for secured communication in telemedicine," World Academy of Science, Engineering \& Technology, Issue 68, p.815, Aug. 2012.

[7] Q. Gu, and T. Gao, "A novel reversible watermarking scheme based on block energy difference for medical images," in the Proc. IEEE Soft Computing and Intelligent Systems (SCIS) and 13th International Symposium on Advanced Intelligent Systems (ISIS),pp. 232 - 237, Nov. 2012.

[8] M. Arsalan, S. A. Malik, A. Khan, "Intelligent reversible watermarking in integer wavelet domain for medical images," The Journal of Systems and Software, 85, pp. 883-894, 2012.

[9] P. Nagarju, R. Naskar, and R. S. Chakraborty, "Improved histogram bin shifting based reversible watermarking," in the Proc. IEEE 2013 International Conf. on Intelligent Systems and Signal Processing, pp. 62-65, 2013.

[10] A. Said, W. A. Pearlman, "A new, fast, and efficient image codec based on set partitioning in hierarchical trees," IEEE Trans. Circuits and Systems for Video Tech., vol. 6, no. 3, pp. 243-250, 1996. 